\title{
Research on Test Method of Self-Propelled Agricultural Machine Based on Virtual Reality
}

\author{
Jinmei Wu*, Han Peng, and Chuangang Wang
}

School of Mechanical Engineering, North China University of Water Resource and Electric Power, Zhengzhou 450045, China

\begin{abstract}
Method: analyze current farm environment, establish 3D virtual scenario of a farm, construct a virtual reality system combining physical engine, mechanical kinematics and dynamics, and simulate whole performance of the self-propelled machine based on virtual reality; Result: the agricultural mechanical system based on virtual reality can accurately capture the realistic road conditions and accurately fit the navigation routes. The climbing and line brake test simulation results of the selfpropelled agricultural machine indicate that the whole traction, speed and vertical shift comply with the actual conditions and validate relation between the speed and shift. Conclusion: the agricultural machine design based on virtual reality is valuable for research on the agricultural modernization, which can reduce design cycle of the agricultural machine, lower design cost of agricultural machines, and improve mechanical performance, so it should be emphasized in industrial production.
\end{abstract}

Keywords: 3D virtual scenario, physical engine, mechanical kinematics, self-propelled agricultural machine, navigation route, virtual reality.

\section{INTRODUCTION}

Agriculture is an industry to product foods and industrial raw materials by cultivating animals and plants. The labor object of the agriculture is the vital animal and plant and the obtained product is the animal and plant. We call departments, which obtain products based on artificial cultivation by using growth and cultivation law of animals and plants, as the agriculture. The agriculture can provide foundational products for supporting country economy construction and development. The agriculture modernization indicates the process and means from traditional agriculture to modern agriculture. The agriculture is armed increasingly with modern industry, modern science technology and modern economy management mode in this process, makes agriculture productivity from the backward traditional agriculture increasingly to modern advanced agriculture in the world. The agriculture modernization can be summarized as "four modernization", namely mechanization, science, irrigation and electrification. The mechanization is ranked as the first position in agriculture modernization [1]. The called agriculture mechanization indicates to replace the manual labor with advanced devices. The mechanical jobs are extensively used at different steps prior to production, in production and after production in order to reduce the physical strength of the labor and improve labor efficiency [2]. With development of science technology, technical requirements for design and development of agricultural machine system become higher and higher. The types of the required agricultural mechanical products are also diversified and work environment is complicated due to diversified terrains and crops in China, so diversity, longer design cycle, randomness of working conditions, and complexity of agricultural mechanical structures of the agricultural mechanical products should be fully considered. How to shorten design time of agricultural machines, deal with diversity of agricultural terrain and crops in China, and optimize design scheme are the common subjects discussed in disciplines such as agriculture and mechanical manufacturing.

The virtual reality (VR) is a new high technology emerging in recent years, which is also called as virtualization technology or artificial environment technology. The virtual reality indicates to generate a 3D virtual world by using PC simulation, provide users with vision, hearing and touch simulation, and make users immersive and observe objects in 3D space truly in time. The VR technology provides an effective means to solve existing problems in development of the traditional agricultural mechanical products, and also reforms methods and ideas in production field [3, 4]. The functions, appearance and performance of mechanical machines can be effectively modeled with VR technology. At same time, a "true" 3D virtual model can be generated. Visualization technology based on VR can be used to observe agricultural production activities of agricultural machines in virtual models, evaluate performance of the agricultural machines, truly realize real-time interaction between human being and agricultural machines, and improve scientific research efficiency and product quality. The VR technology can be used to analyze dynamic performance of the models, replace 
the traditional prototype test with digital manner, reduce development expense and cost of products, and improve product quality and performance [5]. The VR technology can reduce design cycle of the agricultural machines, improve comprehensive utilization rate of the agricultural resources, and provide a "true" platform for education, training and scientific research of agriculture technologies [6]. Based on the above description, this paper starts with physical motion law and dynamic attributes and establishes the virtual model of self-propelled agricultural machines in order to discuss application of the VR technology in the test of the selfpropelled agricultural machines.

\section{COMPOSITION AND SCENARIO CONSTRUCT- ION OF VR TEST SYSTEM FOR SELF-PROPELLED AGRICULTURAL MACHINE}

For virtual test of a self-propelled agricultural machine based on VR, a "realistic" model should be established, namely highly vivid 3D model, which should be highly vivid and highly interactive VR model. To implement a realistic model, the 3D modeling, visual simulation and physical engine are necessary. In addition, it is very vital to establish a vivid virtual scenario [7].

\subsection{Development Environment and Function of VR System}

The VR is a visual interface technology. Establishing a complete Vega Prime development environment is the first step to develop VR. The system software development platform mainly includes development tool VisualStudio. NET, 3D design software Pro/E, UG, 3dsMax, source's physical engine ODE, 3D modeling software package Multigen Creator and visual simulation softwareMultigen Vega Prime. The system is divided into the virtual scenario modeling, VR real-time interactive simulation control and VR physical engine [8].

\subsubsection{Visual Studio. NET Software Development Tool}

It is also called as VSNet and is a frequent visual programming tool. This tool is presented by Microsoft in 2002 and is a development tool based on NET architecture. This architecture combines the powerful function with new technologies, which is used to construct an application with highlighted user experience, realize seamless communication across technology boundary, and support different business flows. This architecture is extensively applied in the system program design, database development, AI and computerassisted design, and can fully embody object-oriented and modular design idea.

\subsubsection{MultiGen Creator Modeling Software}

Creator is a special 3D modeling software for visual simulation and is developed by American MultigenParadigm. It has an open Flight data format optimized for real-time application and provides powerful polygon modeling, vector modeling and precise generation function of large-area terrain as well as multiple professional options and plug-in. This software can efficiently generate real-time 3D (RT3D) database in optimal manner, can closely associate the followed real-time simulation software, and leads in real-time simulation fields such as visual simulation, simulation training, city simulation, interactive game and engineering application and scientific visualization in the world. Multigen Creator is a software package and is a realtime 3D model, which is specially created for visual simulation. Creator can easily realize input, structuring, change, prototype creation and model database optimization. It is not only used for large-scale visual simulation, is but also used to create entertainment game environment. Creator mainly aims at how to generate a geographic environment model such as vivid large-area terrain and landscape and improve practicability of the model, so it is very suitable for making visual simulation of large-scale virtual scenarios. MultiGen Creator is mainly used to model plants, crops, performance test field, paddy field, houses and trees at the virtual test scenarios in the agricultural test system based on VR.

\subsubsection{MultiGen Vega Prime Visual Driving Software}

Vega Prime is the latest real-time 3D VR development tool presented by MultiGen-Paradigm, which features object-orientation, powerful functions, friendly interface and platform compatibility. TheVega Prime real-time 3D VR development tool is extensively applied in the aerospace flight vehicle test, real-time simulation and emulation of industrial mechanical device, real estate visual building display and digital cities. Vega Prime has a powerful rendering function and many software tools are inserted as plug-ins, so it not only realizes very visually vivid 3D virtual environment, but also reduces programming of the source codes and quickly and simply creates, edits and runs complicated real-time 3D simulation software.

\subsubsection{Physical Engine}

The physical engine (open dynamic engine, ODE) computes motion, rotation and collision by assigning true physical attributes to the rigid objects. It is not necessary to use physical engines for each game. Simple "Newton" physics (e.g. acceleration and deceleration) can be implemented via programming or script writing to some extent. The multi-rigid body system dynamics research method is the analysis dynamics method with the Lagrange as the representative. TheLagrange function is established as follows:

$$
\begin{aligned}
& \Gamma\left(x_{1}, x_{2}, \cdots x_{n}, \partial_{1}, \partial_{2}, \cdots \partial_{m}\right)=F\left(x_{1}, x_{2}, \cdots x_{n}\right) \\
& +\sum \partial_{i} G_{i}\left(x_{1}, x_{2}, \cdots x_{n}, \partial_{1}, \partial_{2}, \cdots \partial_{m}\right)
\end{aligned}
$$

$\Gamma(\bullet)$ is the new target function, $F(\bullet)$ is the original function, $G(\bullet)$ is the constraint function and $x_{i}, \partial_{i}$ is the constraint function.

In addition, the vector method, graph theory method, Kane method and variationalmethod with Newton-Euler equation as the representative are the dynamics analysis method of the physical engine.

\subsection{Construction of 3D Virtual Scenario Model}

The virtualscenario plays an important role in VR test system of the agricultural machine. A virtual scenario is created to obtain a true 3D data. Quality of 3D scenario will 
directly affect simulation effect of the system. The established 3D scenario complexity will change with the demand of the agricultural machines. If the established model is too simple, it will affect immersion feeling and fidelity of the simulation environment of the virtual scenario. If the 3D modeling is too complicated, it will affect simulation speed of the virtual scenario, so the key for system failure or success is to establish a $3 \mathrm{D}$ virtual scenario suitable for VR test system of agricultural machine [9].

For the above modeling issue, the virtual scenario is wholly designed via field investigation, data collection and image sampling according to the actual scenario of the test station. The virtual scenario model of the self-propelled agricultural machine includes the terrain scenario model and crop/facility scenario model. The terrain scenario includes the farmland ground, farmland ridge, ditch, complicated obstacles and random ground. The crop/facility scenarios include farmland crop, green plants, public facilities, building and dike water surface. The virtual scenario structure is shown as the Fig. (1).

\subsection{Establishment of Kinematics and Dynamics of Self- propelled Agricultural Machine}

The fidelity of the 3D body structure of the test vehicle in the virtual environment should be considered and the true compliance to physical law in movement of self-propelled agricultural machine should be considered in creation of the self-propelled machine model. The kinematics can analyze mass point motion of the agricultural machine by using the geometric method. The dynamics studies power influences on the machine under the action of external factors (including road conditions and weather). We will simply describe important components of the self-propelled agricultural machine model in the following part, namely navigation path pickup model, engine mathematical model and traction performance dynamics model. For the navigation path pickup model, the navigation path pickup is the key step for virtual realization of visual navigation. Generally the automated navigation line of agricultural machine is extracted according to the features of farmland ridge line, slope, ditch and non-harvested crop edge. The navigation route is simplified as the fitting curve of the short line in the navigation route pick-up model of the agricultural machine, namely the navigation route is a curve, the curve can be processed as short lines. Now the line path is identified by using the Hough transformation, least squares method and template match method. The navigation path pickup algorithm flowchart is shown as the Fig. (2).

Engine's mathematical model: To simplify the model, the throttle openness should meet the control requirement for engine revolving speed and output torque and the heating power process inside the engine is not considered in the agricultural machine virtual test system based on VR. The driving force affecting the agricultural traction performance is identified by the engine performance and ground attachment condition. Based on the research achievements in the reference [7], we know:

$M_{l}=f\left(\alpha, n_{s}\right)-\frac{1}{2} m r^{2} * \omega$

$M_{l}$ is the torque of the engine load, $f()$ is the quasistatic torque mapping of the engine, $\alpha$ is the throttle openness, ns is the engine revolving speed, $\omega$ is the revolving angle acceleration of the engine, $m$ is the flywheel mass and $r$ is the flywheel radius.

The least squares method is used as the model's target function:

$$
F=\sum\left[P_{n}\left(X_{i}\right)-y_{i}\right]^{2}=\sum\left(\sum \lambda_{j} x_{i}^{j}-y_{i}\right)^{2}
$$

Dynamics model of traction performance: The traction feature of the agricultural machine indicates to get the optimal relationship between the sliding revolving speed $\mathrm{S}$, actual revolving speed $\mathrm{V}$, traction power $\mathrm{P}$ and traction $\mathrm{F}$ when the machine stably operates under certain resistance conditions. Notice that the resistance includes the rolling resistance and slope resistance in operation of an agricultural machine and the air resistance and acceleration resistance is ignored.
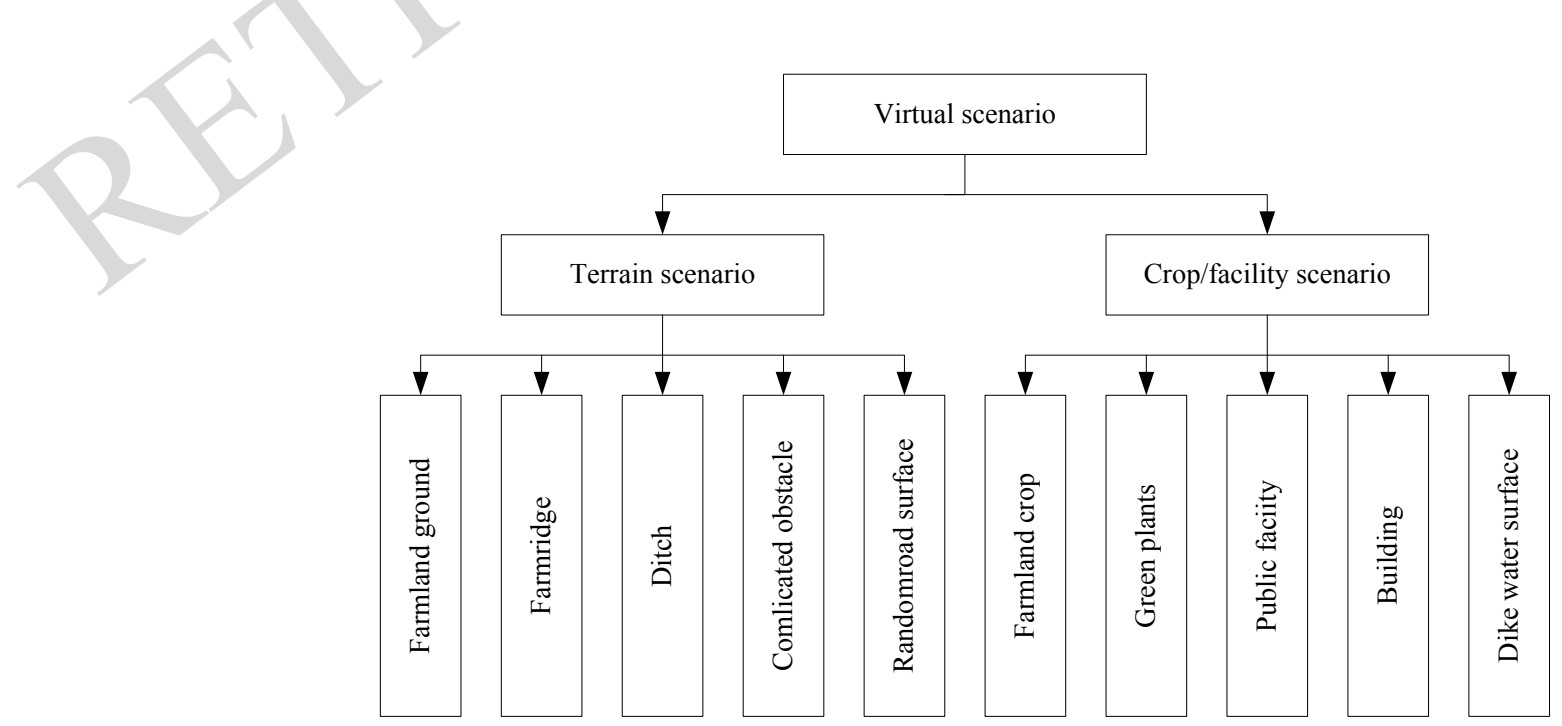

Fig. (1). The virtual scenario structure. 


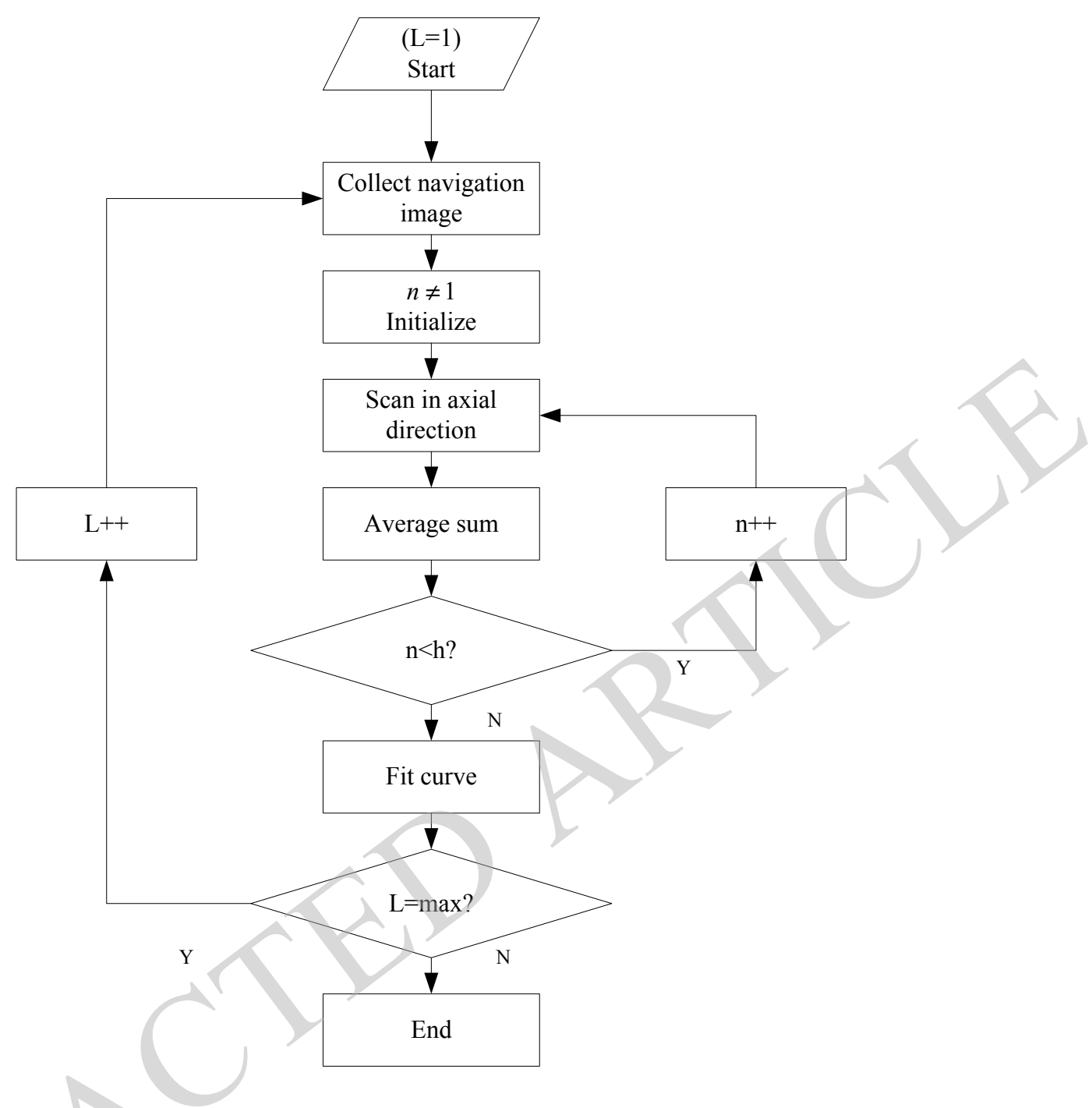

Fig. (2). The navigation path pickup algorithm flowchart.

\section{SYSTEM SIMULATION}

To simulate the VR model in real time, first a complicated mathematical model, geometric model and effective combination of dynamics models should be established. This system uses the hybrid programming method of MATLAB/Simulink and VC. The RTW (RealTime Workshop) extension tool is used to transform the model and the physical engineis used to draw the VR scenario and control the physical parameters of the system. The fitting navigation route of the curve will vary with the navigation fitting model in the automated navigation model simulation of the route. In addition, the navigation results will be restricted by the fitting time. The experiences indicate that the high-order fitting precision is superior to low-order fitting precision. However, the high-order fitting computing time will become longer. This paper fits the navigation routes by using the Hough transformation method and fits the system's navigation route by using the linear, 4order equation and 10-order equation. The fitting results are shown as the Fig. (3).
The Fig. (3) indicates that the Hough transformation method can accurately fit the navigation route and 1-order fitting, 2-order fitting and 10-order fitting can better fit the navigation route. Higher fitting order will bring higher fitting precision. The Fig. (3) indicates that 10-order fitting effect is superior to 4-order fitting effect, but the fitting precision is not boosted much. The fitting time is far higher than 4-order fitting, so the low-order fitting method should be used to fit the navigation route in the VR system simulation.

One main purpose of the agricultural machine virtual test system is to simulate the whole performance of the machine. Based on the structural mechanics and multi-body dynamics theory, the performance of the agricultural machine is analyzed in the created test scenario. The main analysis performances include climbing performance, brake performance, smoothness performance, traction performance, obstacle exceeding or dynamic performance of random road surface. This paper mainly considers relationship between the traction, vehicle speed and vertical shift in simulation of the whole machine and simulation of the brake performance when the whole machine moves in a line. 


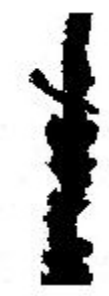

(a) Scanned navigation image

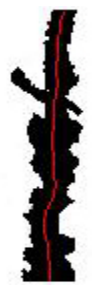

(c) Low-order navigation simulation image

Fig. (3). Fitting results of navigation routes.

The simulation curve of the climbing performance is shown as the Fig. (4).
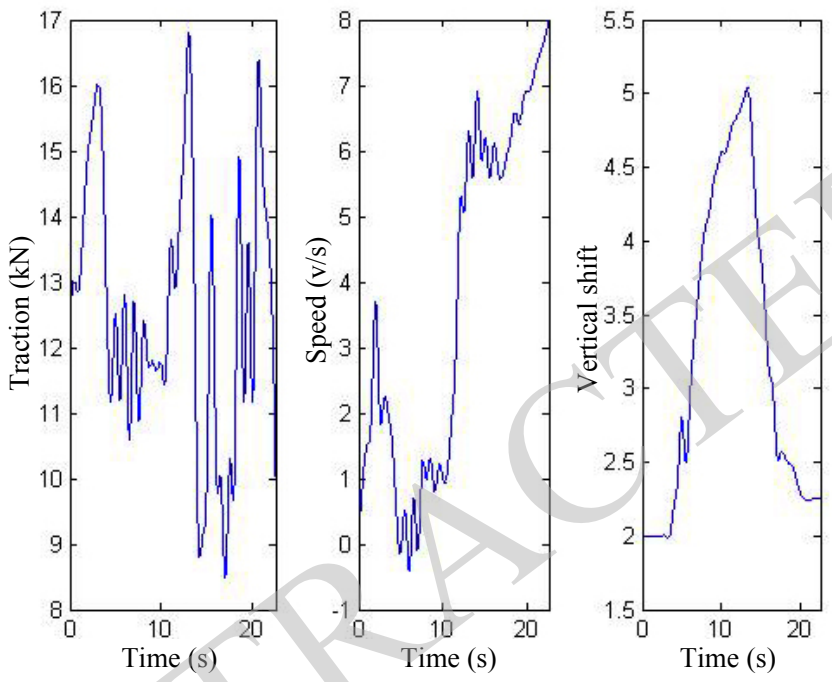

Fig. (4). Simulation curve of climbing performance.

The Fig. (4) shows the performance simulation curve of the agricultural machine for 200 slope, which can intuitively reflect the change relation between climbing traction, climbing speed and climbing vertical shift. The figure indicates that the traction will grow and the speed and vertical shift will decrease when the whole agricultural machine is climbing. When the whole agricultural machine descends, the speed and vertical shift will grow. The following Fig. (5) shows the simulation results of the brake system when the whole agricultural machine moves in a line.

The Fig. (5) indicates that the whole agricultural machine speed grows at an acceleration within $0-5 \mathrm{~s}$. At this time, the shift will increase. When the speed reaches maximum and keeps stable, the shift increase speed will become high. When the speed reduces, the shift increase speed will slow down. The Fig. (5) indicates that the shift is integral of the speed in the time.

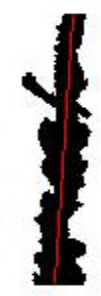

(b) Linear navigation simulation image

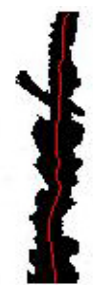

(d) High-order navigation simulation image
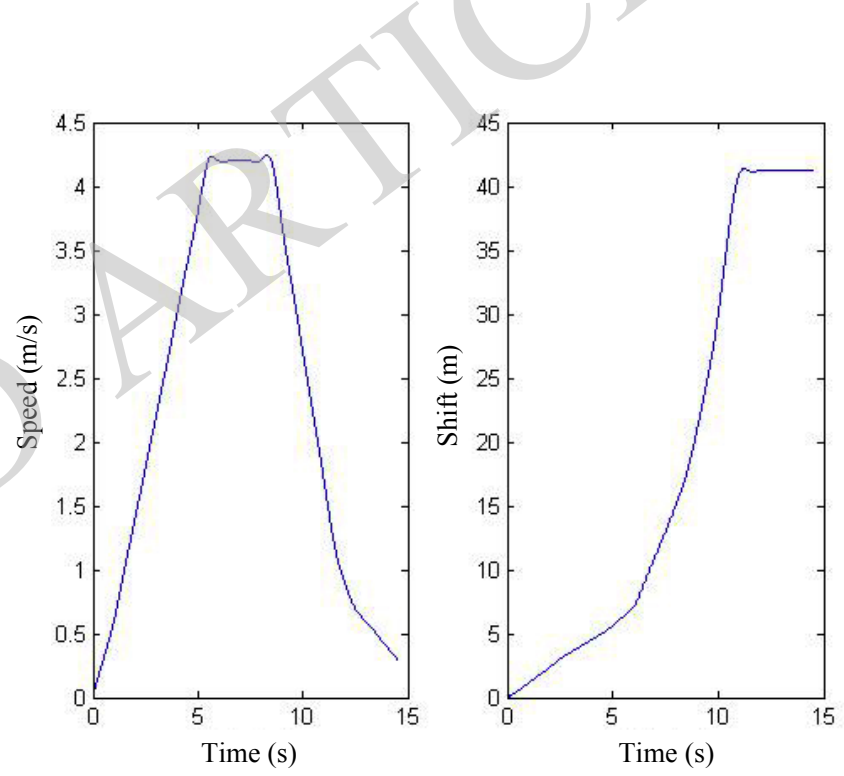

Fig. (5). Simulation line of line brake.

\section{CONCLUSION}

This paper mainly studies application of the VR in the design scheme of agricultural machines, establishes a 3D virtual scenario model based on analysis on the existing farmland environment and actual conditions of the farmland, establishes the VR system combining physical engine, kinematic of machinery and dynamics based on it, and performs performance simulation of the whole agricultural machine. The simulation results indicate that the VR route navigation model can accurately capture actual road conditions and can accurately navigate the route. The climbing simulation of a self-propelled agricultural machine indicates that the whole traction, speed and vertical shift will affect each other, which meets the actual conditions. The line brake test results validate that the shift is integral of the speed in the time, which complies with the realistic requirements.

The mechanical design involves extensive range, features big workload and complicated types in agricultural 
modernization. This paper only studies the self-propelled agricultural machines. The fact proves that this system is only suitable for research on the small agricultural test farmland. For large and complicated agricultural terrain and different crop farmlands, the practicable performance of this research will reduce much. Nevertheless, it is certain that the agricultural machine design based on VR is very valuable for agriculture modernization. This design method can reduce the cycle of the agricultural machines, reduce the mechanical design cost and improve mechanical performance to much extent. It is expected that application of the agricultural machine design based on VR is emphasized due to discussion and research in this paper.

\section{CONFLICT OF INTEREST}

The author confirms that this article content has no conflict of interest.

\section{ACKNOWLEDGEMENTS}

Declared none.

\section{REFERENCES}

[1] W. $\mathrm{Xu}$, "Domestic and foreign research conditions and development of VR technology", Modern Business Trade Industry, no. 2, pp. 279-280, 2009.

[2] Y. W. Yuan, "Research on intelligent equipment virtual simulation technology of digital agriculture", M.S. thesis, Beijing: Chinese Agricultural Mechanization Science Research Academy, 2010.

[3] H. Yin, Z. D. Wang, and J. H. Xu, "Battlefield scenario simulation based on Creator/Vega", Journal of PLA Science and Engineering University (natural science edition), vol. 6, no. 2, pp. 137-142, 2005.

[4] Z. X. Guo, "Research on self-propelled tobacco leaf cropper navigation technology based on machine vision", M.S. thesis, Zhengzhou: Henan Agricultural University, 2013.

[5] S. F. Chen, Z. L. Li, T. Shen, and F. Liu, "Design and test research on variant fertilization hydraulic system", Hydraulics and Pneumatics, no. 12, pp. 102-105, 2011.

[6] V. Leemans and M. F. Destain, "A Computer-vision Based Precision Seed Drill Guidance Assistance", Computers and Electronics in Agriculture, vol. 59, pp. 1-12, 2007.

[7] V. Subramanian, T. F. Burks, and A. A. Arroyo, "Development of Machine Vision and Laser Radar Based Autonomous Vehicle Guidance Systems for Citrus Grove Navigation", Computers and Electronics in Agriculture, vol. 53, no. 2, pp. 130-143, 2006.

[8] Y. Zang, Z. X. Zhu, and Z. H. Song, "Establishment of agricultural equipment virtual test system platform”, Agricultural Machinery Journal, vol. 41, no. 9, pp. 70-74, 2010.

[9] R. H. Zhang and G. P. Zheng, "Design and implementation of collision detection in real-time drawing of high-fidelity dynamic terrain", Computer application, vol. 26, no. 11, pp. 2773-2774, 2006.

[10] H. J. Zhang, J. G. Sun, and X. J. Liu, "Research progress of largescale 3D terrain visualization algorithm", Computer Science, vol. 34 , no. 3, pp. 10-16, 2007.

Received: December 8, 2014

Revised: December 15, 2014

Accepted: December 16, 2014

(C) Wu et al.; Licensee Bentham Open.

This is an open access article licensed under the terms of the Creative Commons Attribution Non-Commercial License (http://creativecommons.org/licenses/by-nc/3.0/) which permits unrestricted, non-commercial use, distribution and reproduction in any medium, provided the work is properly cited. 